\title{
The value of evidence synthesis
}

\author{
Science is a cumulative enterprise, and systematic evidence synthesis is invaluable for appraising what is known \\ and what is not known on a specific research question. We strongly encourage the submission of systematic \\ reviews and meta-analyses to Nature Human Behaviour.
}

S ystematic evidence synthesis involves the combination of information from multiple studies that have addressed the same research question, to extract a summary understanding of what is known at a specific point in time about the specific question. Over recent decades, evidence synthesis has grown into a discipline in its own right, with substantial effort invested in developing methods, tools, statistical techniques, and reporting standards for high-quality syntheses of evidence.

Systematic evidence synthesis has a long history in the health sciences. The earliest example of a systematic review was written by James Lind in 1753, who produced a treatise on scurvy based on the then-available published evidence on the disease ${ }^{1}$. In more recent times, reliable information from research on the benefits and harms of specific interventions has become absolutely critical for evidence-based medicine, and systematic reviews and meta-analyses of randomized controlled trials occupy the pinnacle of hierarchies of evidence quality in clinical research. Although systematic evidence synthesis has a more recent history in the social sciences, it is now a cornerstone of assessing research evidence on societally relevant questions to inform policy.

Across the numerous fields in which it is now widely practiced, systematic evidence synthesis has a crucial role in distilling not just what is known, but also how much confidence we can have in the existing evidence. In his treatise on scurvy, Lind noted that "before the subject could be set in a clear and proper light, it was necessary to remove a great deal of rubbish." Assessing the quality of evidence from individual studies is one of the most important functions of systematic reviews: rigorously performed systematic reviews evaluate risk of bias in individual studies and provide a snapshot of issues that
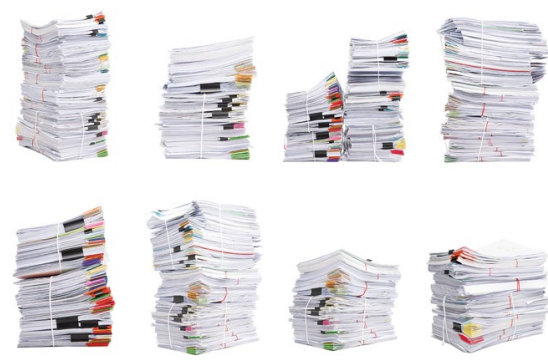

Credit: JATURON RUAYSOONGNERN / ALAMY STOCK PHOTO

undermine confidence in what is known, such as design flaws, small sample sizes, lack of preregistration or discrepancies between protocols and published studies, and inconsistent or incomplete reporting.

Evaluation systems and funding bodies frequently place higher value on original research rather than evidence synthesis, especially outside clinical domains.

However, as the ongoing pandemic has amply shown, evidence synthesis may be one of the most valuable contributions the scientific community can provide to policy and other decision-makers. At Nature Human Behaviour, we believe strongly in the value of systematic evidence synthesis: high-quality evidence syntheses are on par with original research and can constitute contributions of outstanding scientific and applied significance.

When we evaluate systematic reviews (and meta-analyses), we are looking for topics of broad significance in productive areas of research that would benefit from systematic synthesis of the available evidence. For systematic reviews in broad, productive areas within our scope, we prioritize for peer review and publication manuscripts that exhibit high methodological rigour. We strongly encourage authors to register prospectively their systematic reviews on PROSPERO, the international prospective register of systematic reviews, and ask that they report any deviations from their preregistered protocol in the submitted manuscript. Regardless of whether a systematic review was preregistered or not, we require that authors follow best practices in reporting and ask that they complete and submit the Preferred Reporting Items for Systematic Reviews and Meta-Analyses (PRISMA), or the most suitable PRISMA extension, along with their manuscripts. Manuscripts should also be accompanied by a PRISMA flow diagram that transparently presents the number of records identified in the course of searching the literature, which records were included and excluded, and the reasons for exclusions.

This issue features one such contribution by van Agteren and colleagues $^{2}$, who synthesized the vast literature of psychological interventions aimed at improving mental states of wellbeing. Appraising the efficacy of these interventions for clinical and non-clinical populations is critical for guiding health care and for identifying where the quality of evidence is not yet sufficient to draw reliable conclusions. We hope to publish many more high-quality evidence syntheses in the future and encourage you to submit such work for publication in our pages.

Published online: 20 May 2021

https://doi.org/10.1038/s41562-021-01131-7

\footnotetext{
References

1. Lind. J. A treatise on the scurvy. In three parts. Containing an inquiry into the nature, causes, and cure, of that disease. Together with a critical and chronological view of what has been published on the subject. (Sands, Murray and Cochran for A. Kincaid and A. Donaldson, 1753)

2. van Agteren, J., Iasiello, M. \& Lo, L. et al. A systematic review and meta-analysis of psychological interventions to improve mental wellbeing. Nat. Hum. Behav. https://doi.org/10.1038/s41562-02101093-w (2021)
} 